Available online at:

https://acta-acustica.edpsciences.org

\title{
A laboratory study for occupational safety and health on the structure of airborne ultrasound fields
}

\author{
Robert Schöneweiß*, Christoph Kling, and Christian Koch \\ Physikalisch-Technische Bundesanstalt (PTB), Bundesallee 100, 38116 Braunschweig, Germany
}

Received 27 January 2020, Accepted 28 July 2020

\begin{abstract}
While exposure to airborne ultrasound is increasing in occupational contexts and in public and private spaces, existing demand for reliable and traceable determination of exposure to sound with frequencies above $16 \mathrm{kHz}$ cannot currently be satisfied due to a lack of adequate measurement devices and procedures adapted to the specifics of airborne ultrasound. So that this study may serve as a first step for the development of a novel measurement procedure, its aim is to create a comprehensive database of the structures of airborne ultrasound fields present in occupational contexts. Based on this, the limitations of measurement procedures commonly used in the audible frequency range are clarified and the structural characteristics of airborne ultrasound fields investigated. This paper presents a laboratory study of the structure of the airborne ultrasound field of an ultrasonic welding machine, which can be considered a representative occupational source of airborne ultrasound. For this study, the technical and procedural requirements of a measuring system are derived and used to set up and calibrate a measuring system for three-dimensional, high spatial resolution scans of sound pressure levels in the laboratory. The measurement results reveal complex, extensive and very fine-structured interference patterns, some of which have sound pressure levels of up to $138 \mathrm{~dB}$ (re $20 \mu \mathrm{Pa}$ ).
\end{abstract}

Keywords: Airborne ultrasound, Measuring system, Spatial scanning of sound pressure levels, Sound field mapping, Ultrasonic noise

\section{Introduction}

Exposure to airborne ultrasound is increasing in occupational contexts and in public and private spaces [1]. Sources of airborne ultrasound can be found in areas as diverse as laboratory and medical technology, measurement devices, and industrial applications such as cutting, welding, and cleaning. Today, ultrasound sources that emit airborne ultrasound due to their primary action principle or as a side effect are already widely used and increasing in use. However, research on how humans perceive airborne ultrasound or are otherwise impacted by it, as well as on the development of measurement devices and procedures, has not matched the pace at which ultrasound technology has proliferated. In most cases, the validation of measurement devices and measurement procedures is limited to the audible frequency range, particularly with regard to occupational safety and health. It is at best questionable whether a simple application of measurement procedures commonly used in the audible frequency range to ultrasonic frequencies is effective. The lack of reliable measurement

*Corresponding author: robert.schoeneweiss@ptb.de procedures adapted to the specific characteristics of airborne ultrasound impedes the investigation of many questions concerning the health risk caused by exposure to airborne ultrasound [2]. The unavailability of measurement devices and procedures represents an obstacle to research, resulting in inadequate scientific data and unreliable maximum permissible sound pressure levels [1].

This paper presents three-dimensional, high spatial resolution measurements of the sound field of a representative occupational source of airborne ultrasound that were carried out within the scope of the research project titled "Ears II - Metrology for modern hearing assessment and protecting public health from emerging noise sources" [3]. The organization of this paper closely follows the steps taken in the development of the measuring system used in the above research project. First, technical and procedural requirements for a measuring system were derived. Based on these requirements, a measuring system was set up and subsequently used for extensive measurements of the airborne ultrasound field of an ultrasonic welding machine. Laboratory measurements were conducted to obtain a comprehensive database by means of which the restrictions of measurement devices and procedures commonly used in the audible frequency range can be analyzed. The results 
verified that the measuring system fulfilled the specified requirements, justified the creation of a novel measurement procedure to determine occupational exposure to airborne ultrasound, and served as the basis for the development of a measurement procedure. Moreover, the investigations allowed knowledge to be gained about which instruments were appropriate for measuring airborne ultrasound. Measuring airborne ultrasound fields reliably is a vital prerequisite for further investigations, since quantifying ultrasound exposure is the prerequisite for all consecutive activities in occupational safety and health.

Investigating ultrasound fields in liquids (for example, medical ultrasonic fields) represents an additional problematic issue in ultrasound field measurement. For liquids, very different types of field-scanning techniques that use different types of hydrophones and noninvasive techniques have been published and established [4]. Based on these techniques, acousto-optic methods such as optical diffraction tomography [5] and optical heterodyne probing [6] have been adapted for the investigation of airborne ultrasound fields. This paper presents direct microphone measurements under laboratory conditions using a measuring system designed to reduce the complexity and costs of the experimental setup. This measuring system allowed sound pressure in the sound field to be measured directly by means of traceable measuring instruments, although it does not offer the completely non-disturbing measurements that optical techniques provide.

Within the scope of this paper, ultrasound is defined as sound having frequencies above $16 \mathrm{kHz}$. The reference value of all sound pressure level quantities mentioned in this paper is $20 \mu \mathrm{Pa}$. All relevant datasets generated and/or analyzed in this study have been deposited at a public research data repository $[7,8]$.

\section{Measuring system}

\subsection{Requirements}

Although no strict, clear threshold between audible sound and ultrasound exists in the realm of physics, differences in the characteristics of audible sound and ultrasound become evident when comparing sound from separate frequency ranges. Ultrasonic frequencies, i.e. frequencies above $16 \mathrm{kHz}$, correspond to wavelengths of approximately $21 \mathrm{~mm}$ and below in air at a temperature of $20^{\circ} \mathrm{C}$ assuming a speed of sound of $343 \mathrm{~ms}^{-1}$. Thus, ultrasound has considerably smaller wavelengths than audible sound and, consequently, different characteristics. Compared to audible sound, the emission of ultrasound is more directional, and ultrasound fields have finer interference patterns. Moreover, attenuation due to different kinds of dissipation ([9], pp. 161-163; [10]) increases with the square of the frequency and is therefore more pronounced in the ultrasonic frequency range than attenuation in the audible sound range. As a response to these properties, the following five requirements for measurements and corresponding setups have been established:

\subsubsection{Frequency range}

It is known that the acoustic emission of ultrasonic devices is dominated by tonal components at the working frequency as well as by components at the corresponding higher harmonics at integral multiples of the working frequency, subharmonics at integral divisors of the working frequency and ultraharmonics at rational multiples of the working frequency. Since the frequency range of $16-40 \mathrm{kHz}$ [11] covers the working frequencies of most industrial or laboratory ultrasound sources, it is preferable that the measuring chain have a flat frequency response in the frequency interval of 6-90 kHz under free-field conditions. This allows the signal to be analyzed starting with the $8 \mathrm{kHz}$ one-third octave band and ending with the $80 \mathrm{kHz}$ one-third octave band. To this end, the first subharmonic of the lowest frequency and the first harmonic of the highest frequency of the desired range of working frequencies are analyzed.

\subsubsection{Spatial resolution}

For sound sources that have larger dimensions than the wavelength they emit, a pronounced near field forms around the source. The near field is a subarea of the sound field that is characterized by a complex interference pattern of distinctly localized sound pressure minima and maxima. As ultrasound has small wavelengths in the millimeter range, while sources of airborne ultrasound are typically larger, this effect applies to the majority of ultrasound sources. Like the wavelength of ultrasound, it is assumed that the interference patterns of ultrasound have spatial structures in the millimeter to sub millimeter range. As a consequence of the small wavelength of ultrasound, even small obstacles in the propagation path of ultrasonic waves that differ in their specific acoustic impedance from the propagation medium can cause reflection and scattering. As the resulting secondary waves interfere with other waves, a more complex interference pattern is very likely to occur for this reason as well. Moreover, ultrasonic sound emissions are often monofrequent or at least have a tonal frequency characteristic. Therefore, the resulting interference patterns have a high interference visibility. To resolve this heterogeneous three-dimensional structure, the measuring system must either allow measurements to be taken at different positions or scanning measurements with a high spatial resolution to be performed. The spatial resolution is determined both by the step size of the scan and the size of the microphone diaphragm. Following the sampling theorem, the step size must be at least half the size of the structure to be resolved. Assuming the size of these structures is in the range of the minimal wavelength of the ultrasound under investigation, the step size of the measuring system should preferably be better than $1.9 \mathrm{~mm}$, which is half the wavelength corresponding to ultrasound with a frequency of $90 \mathrm{kHz}$. As the structure of the interference patterns occurring may be even smaller, especially in the near field, a smaller step size is preferable. The required size of the microphone diaphragm was estimated by transferring 
measurement requirements from a standard for measuring ultrasonic fields with hydrophones [12]. According to IEC $62127-1$, the effective radius of the sensor should preferably be equal to or smaller than one quarter of the wavelength of the ultrasound under investigation. For ultrasound with a frequency of $90 \mathrm{kHz}$, the effective radius is $1 \mathrm{~mm}$. The smaller the microphone diaphragm is, the smaller the area is over which the sound pressure is averaged. This enables a higher spatial resolution and minimizes the contribution to the instrumental measurement uncertainty.

\subsubsection{Linearity and dynamic range}

A survey on "noise exposure at ultrasound-related industrial workplaces and public sites" [13] carried out by Wolff et al. revealed that Z-weighted peak sound pressure levels up to $155 \mathrm{~dB}$ may occur. An "analysis of the noise exposure and the distribution of machine types at ultrasound-related industrial workplaces in Germany" [11] carried out by Ullisch-Nelken et al. further supports the need for measurements at such high sound pressure levels. Therefore, the linear response of the measuring system should preferably extend to at least this value for the peak sound pressure level. Furthermore, the results of both publications suggest a lower limit of the measuring instrument's dynamic range of approximately $60 \mathrm{~dB}$. A large dynamic range makes it possible to detect the expected sound pressure extrema occurring due to the high interference visibility.

\subsubsection{Signal-to-noise ratio}

The components of the electro-acoustic measuring chain should preferably ensure a suitable signal-to-noise ratio (SNR) in the measuring interval of approximately $20 \mathrm{~dB}$. Assuming incoherent noise, this corresponds to a contribution to the instrumental measurement uncertainty of less than $0.1 \mathrm{~dB}$. Therefore, low inherent noise and comparatively high sensitivity of the microphone cartridge are equally important.

\subsubsection{Traceability}

Particularly in the context of occupational safety and health, precise measurement results are essential. Moreover, collaboration with several research partners requires metrological comparability of measurement results, and both require that the metrological traceability of measurement results be established via a metrological traceability chain. To this end, the measuring system must be calibrated. Moreover, the measuring system should preferably be equipped with a coordinate-measuring instrument to link the measuring points to the ultrasound source within the same coordinate system.

In the ultrasonic frequency range, the dimensions of the microphone membrane and the wavelength are in the same order of magnitude. Here, an incident wave with a small wavelength that impinges obliquely is detected at the microphone membrane with a different phase. The pressure integrated over the membrane, and thus the sensitivity of the microphones, are more angle-dependent than in the audible sound frequency range. Even if the influence of the directivity pattern on the measurement results cannot be corrected, it should at least be quantified and considered as a contribution to the instrumental measurement uncertainty.

The phenomena concerning propagation and absorption mentioned above have a strong dependence on the conditions of the atmospheric environment (i.e. humidity, temperature, and pressure) [14-17]. Because this also applies to the measuring system, these environmental conditions must be maintained and measured precisely. Thus, the conditions of measurement are well known and their influence on the measurement repeatability and the intermediate measurement precision can be minimized. All these procedures are necessary to enable good measurement reproducibility.

\subsection{Implementation}

A measuring system that fulfilled the requirements formulated above was designed and set up with the following specifications.

\subsubsection{Scanning unit}

The hardware of the scanner unit was a customized three-axis Isel computerized numerical control (CNC) machine in gantry configuration with a high moving bridge. Its setup is shown in Figure 1a. It consisted of an aluminium frame with dimensions of $2.30 \mathrm{~m} \times 2.45 \mathrm{~m} \times$ $2.40 \mathrm{~m}$ (width $\times$ depth $\times$ height) and top-mounted linear units with Isel LES4 spindle drives. In order to describe the scanning positions and movements of the scanner, coordinate axes were defined that were parallel to the linear units of the scanner. These axes spanned a three-dimensional right-handed Cartesian coordinate system. The coordinate axes and the spatial directions of the scanner were assigned as follows: the $x$-axis designated the width, the $y$-axis designated the depth and the $z$-axis designated the height. Each scanner axis was driven directly (z-axis) or via a toothed belt ( $x$ - and $y$-axis) by an individual two-phase Isel MS 200 HT-2 stepper motor. The step angle of $1.8^{\circ}$ of the stepper motors [18] and the pitch of $5 \mathrm{~mm}$ of the ball screws ([19], p. 11; [20], p. B-38) produced a linear step size of $25 \mu \mathrm{m}$. Reference switches at the end of each linear unit defined the reference point of the system and thus the origin of the coordinate system. A $2.20 \mathrm{~m}$ rod was mounted on the carriage of the scanner in the $z$-direction.

Due to the expected high spatial variation of the ultrasound fields, it is necessary to acquire numerous measuring points to characterize the sound field with sufficient spatial resolution. Since these measurements are time-consuming, the measuring system was designed as a multi-channel device for simultaneous measurement at several measuring points. To this end, a clamp for four $1 / 4^{\prime \prime}$ microphones, which is shown in Figure 1b, was mounted on a coupling at the lower end of the rod. The microphones were aligned as a line array that could be mounted in two ways: within the $x y$-plane with the microphones aimed in the 


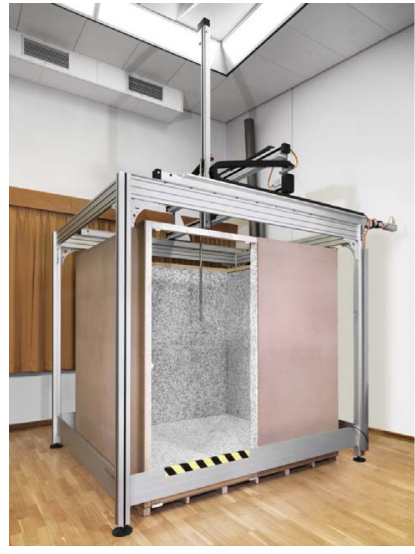

(a)

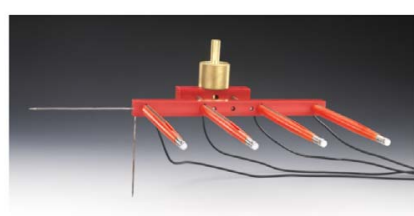

(b)
Figure 1. Components of the measuring system. (a) Scanning unit of the measuring system with an integrated free-field environment. (b) Microphone array with four $1 / 4^{\prime \prime}$ microphones and two mechanical probes for tactile coordinate measurements.

$-y$-direction, which will be referred to as the standard orientation, and within the $y z$-plane with the microphones aimed in the $-z$-direction, which will be referred to as the downgraded orientation. The clamp consisted of turned parts made of Trovidur EC-N, an unplasticized hard polyvinyl chloride (PVC-U). The microphones were clamped in laterally slotted hollow cylinders with thin walls, which enabled the microphones and their connecting cables to be placed inside the clamp. The reflection face was minimized by the thin-wall design in combination with the outer shape of the clamp, as well as by placing the microphones and their connecting cables inside the clamp. The individual microphone clamps were mounted at a distance of $60 \mathrm{~mm}$ to each other on a small cross rail with a cable feedthrough to the back.

For tactile coordinate measurements of objects inside the scanner unit, two mechanical probes were mounted on the cross rail of the microphone clamp. The probes were $100 \mathrm{~mm}$ long, perpendicular to each other and, based on the standard orientation of the clamp, orientated in the $x$-direction and the $-z$-direction, respectively.

For observation of the interior of the free-field environment during positioning processes and measurements, a camera system was installed. For the top view, a Logitech BRIO Ultra HD webcam was mounted at the upper end of the rod. For binocular vision, two Microsoft HD 3000 LifeCams were placed in two internal corners of the freefield environment.

The electrical power supply and the control of the three two-phase stepper motors were realized by means of a multiple-axis Isel iMC-P1-3 controller, which was connected to a PC. Using the manufacturer's library of control commands, the three-dimensional movements of the cantilever were remote-controlled via a purpose-built software program with a graphical user interface (GUI) implemented in-house using Visual Basic 2015.

For monitoring the atmospheric environment, a basic Ahlborn ALMEMO 2490-1 measuring instrument with an
FHAD 46-C2 digital sensor for temperature, atmospheric humidity and atmospheric pressure was used.

\subsubsection{Electro-acoustic measuring chain}

1/4" GRAS 40BF microphone cartridges were chosen for the measuring system since they represent a reasonable compromise in fulfilling all but one of the requirements formulated in Section 2.1. According to the manufacturer's data [21], they have a flat frequency response $( \pm 2 \mathrm{~dB})$ in the frequency interval of $4 \mathrm{~Hz}$ to $100 \mathrm{kHz}$ under free-field conditions, a linear response up to $172 \mathrm{~dB}$, a dynamic range lower limit due to thermal noise of $30 \mathrm{~dB}$ (A-weighted) and a sensitivity of approximately $4 \mathrm{mV} \mathrm{Pa}^{-1}$. Further advantages of $1 / 4^{\prime \prime}$ microphone cartridges are the fact that they have been used extensively and their availability. The strict criterion of an effective radius of the microphone diaphragm of $1 \mathrm{~mm}$ cannot be satisfied. This strict criterion is satisfied for $1 / 4^{\prime \prime}$ microphone cartridges, which have a radius of $2.95 \mathrm{~mm}$, for frequencies up to $29 \mathrm{kHz}$. However, as mentioned in IEC 62127-1, this criterion may be relaxed under two conditions: measurements must be performed in the far field; and a contribution to the instrumental measurement uncertainty must be considered to accommodate for the biased measuring signal, which is a convolution of the undisturbed signal and an averaging influence of the microphone.

The advantages of the smaller size of $1 / 8^{\prime \prime}$ microphone cartridges are their higher spatial resolution and reduced influence of the microphone on the sound field due to reflection and diffraction. However, even with microphone cartridges of this size, the strict criterion of IEC 62127-1 cannot be satisfied, since the cartridge radius is approximately $1.5 \mathrm{~mm}$ [22]. Furthermore, these advantages are limited by the fact that no $1 / 8^{\prime \prime}$ preamplifiers are available. Instead, adaptors for $1 / 4^{\prime \prime}$ or $1 / 2^{\prime \prime}$ preamplifiers are used, even though they enlarge the reflection face. Moreover, $1 / 8^{\prime \prime}$ microphone cartridges are usually optimized to have a flat frequency response in a pressure field instead of a free field and the lower limit of the dynamic range is typically $52 \mathrm{~dB}$ ( $A$-weighted) [22], which implies a low SNR of less than $20 \mathrm{~dB}$ for sound pressures near the lower limit of the desired measuring interval.

The electro-acoustic measuring chain consisted of four $1 / 4^{\prime \prime}$ externally polarized free-field measurement microphones of the GRAS 40BF condenser type, four 1/4" GRAS $26 \mathrm{AC}$ preamplifiers, two GRAS $12 \mathrm{AQ}$ power modules and an Ono Sokki CF-9400 FFT analyzer connected to a PC. A schematic representation of a single channel of the electro-acoustic measuring chain is shown in Figure 2. Measurements were performed with no protecting grids mounted on the microphones.

Each of the GRAS 12AQ power modules was used to provide a polarization voltage of $200 \mathrm{~V}$ for two microphone cartridges and to power the corresponding preamplifiers with a supply voltage of $\pm 60 \mathrm{~V}$. For the power modules, a filter with a linear response and a high-pass filter of $0.2 \mathrm{~Hz}$ was chosen [23]. The gain was set to $0 \mathrm{~dB}$. 


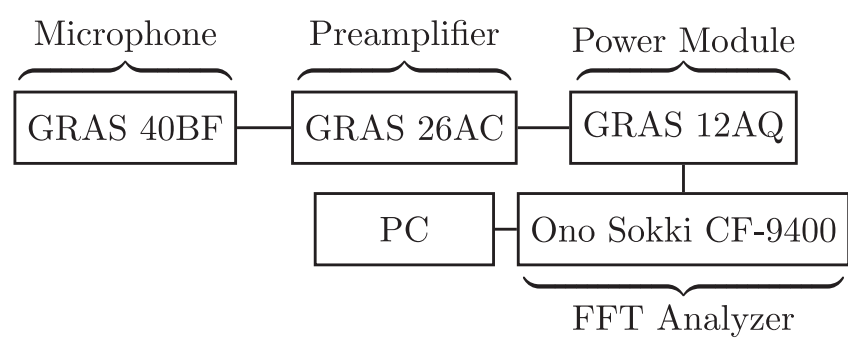

Figure 2. Schematic representation of a single channel of the electro-acoustic measuring chain for measuring the sound pressure.

\subsubsection{Signal processing}

The electrical microphone signals of the four microphones, in terms of the voltage $U$ taken from the output of the GRAS 12AQ power modules, were measured with the four-channel Ono Sokki CF-9400 FFT analyzer for each microphone individually. The signal voltage was sampled with a sample frequency of $256 \mathrm{kHz}$ to cover the frequency range of $0-100 \mathrm{kHz}$ with a fixed oversampling factor of 2.56 . The discretized signal was converted via a fast Fourier transform with a size of $16384=2^{14}$ using a Hann window. The square of the absolute value of the discrete Fourier transform of the signal $|\mathcal{F} U|^{2}$ was represented as level re $1 \mathrm{~V}^{2}$ in the frequency domain by 6401 frequency components, which equals a frequency resolution of $15.625 \mathrm{~Hz}$. This representation, as well as the representation after conversion to sound pressure levels, will be referred to as a periodogram. To avoid aliasing, a digital anti-aliasing low-pass filter cuts signals with frequencies higher than half of the sampling frequency. For each scanning position, the signal was measured up to 31 times, converted via FFT, averaged, and saved with the corresponding metadata. Depending on the type of signal to be measured, different types of averaging were used. For time-independent signals, an exponential averaging process was used to compensate the mechanical shaking of the microphone array induced by the scanner moving to a new scanning position. Since the decay of the oscillation is an exponential process, the average was calculated with an exponentially increasing weighting of the measurement results. For time-dependent signals, the average was calculated in terms of the arithmetic mean of the measurement results.

The measuring data was further processed as an offline analysis with a purpose-built algorithm using MATLAB R2018b (9.5.0.944444; 64-bit). The algorithm was used to import the measuring data of all scanning positions of a measurement and to convert the measuring data into $Z$-weighted equivalent continuous sound pressure levels $L_{p, Z \text {,eq } T}$ using the calibration data acquired. Based on this processed data, the sound pressure levels were plotted for single frequency components or as band sound pressure levels for defined frequency bands.

\subsubsection{Setup}

The scanning unit was set up in a laboratory at the Physikalisch-Technische Bundesanstalt (PTB). The measuring instruments and the controlling PC were placed in a separate control room to minimize the influence on measurements and to protect the staff from ultrasonic noise.

An open-top free-field environment was set up inside the scanning unit using soni PROTECT $\mathrm{R}$ sound insulation panels with a thickness of $50 \mathrm{~mm}$. These panels were made from open-cell foam based on BASF Basotect melamine resin. To avoid reflections, sound insulation panels were mounted on the rod and moving bridge of the scanning system. The resulting free-field environment, as shown in Figure 1a, had dimensions of $1.9 \mathrm{~m} \times 1.9 \mathrm{~m} \times 2 \mathrm{~m}$ (width $\times$ depth $\times$ height). Inside the free-field environment, a desk with dimensions of $1.60 \mathrm{~m} \times 0.72 \mathrm{~m} \times$ $0.80 \mathrm{~m}$ (width $\times$ depth $\times$ height) was mounted flush with one of the walls and covered with a felt layer. This desk served as the substructure of the ultrasound source.

\section{Calibration}

The four microphone channels of the electro-acoustic measuring chain were calibrated separately with traceability to the national standard at PTB. The free-field sensitivity of the microphones at a $0^{\circ}$ incidence angle was determined in the frequency range from $0.25 \mathrm{kHz}$ to $100 \mathrm{kHz}$ with a frequency resolution of $1 \mathrm{kHz}$ using two primary calibrated microphones as transfer measurement devices for a substitution measurement method. The frequency response of the remaining electrical measuring chain was calibrated at the same frequencies. Level linearity in the frequency range considered was shown for all sound pressure levels occurring. The final frequency response of the acoustical and electrical calibration for the four measuring chains was obtained using a $\mathcal{C}^{2}$ interpolating cubic spline and is shown in Figure 3.

The directivity patterns of the microphones were quantified to estimate the corresponding influence on the measurement results. To this end, the microphones were insonified by a loudspeaker with a constant radial distance $r=52 \mathrm{~cm}$ from different directions of sound incidence by varying the polar and azimuthal angles. The positions of the loudspeaker had a distance on the sphere of $3 \mathrm{~cm}$ for

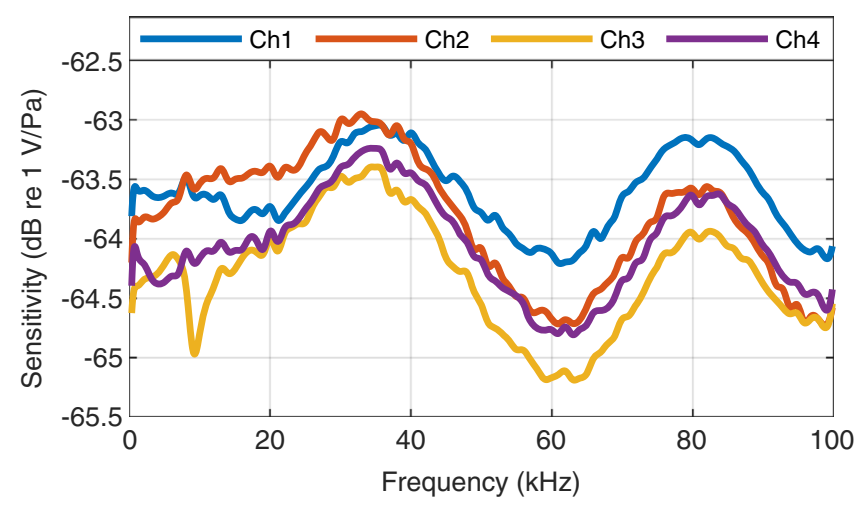

Figure 3. Free-field sensitivity of the four channels of the electro-acoustic measuring chain, denoted as "Ch1" (channel 1) to "Ch4" (channel 4 ), at $0^{\circ}$ incidence angle in the frequency range from $0.25 \mathrm{kHz}$ to $100 \mathrm{kHz}$. 


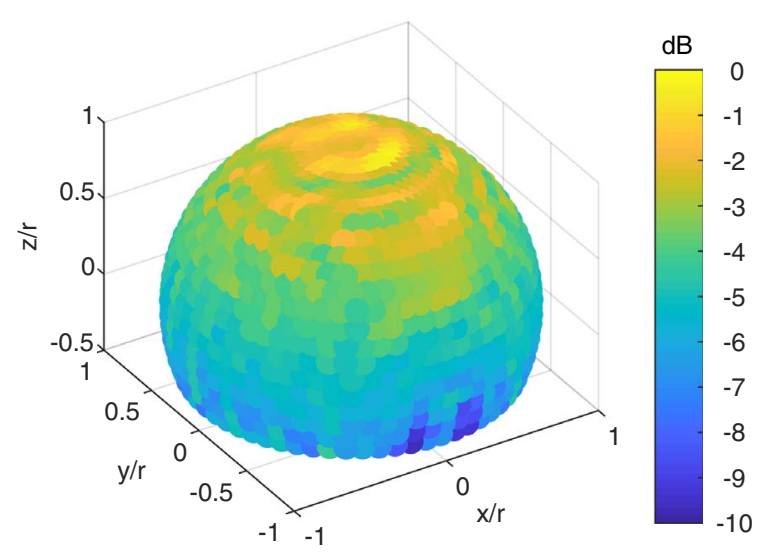

Figure 4. Directivity pattern at $20 \mathrm{kHz}$ of a GRAS $40 \mathrm{BF}$ microphone cartridge. The angle-dependent sensitivity is normalized to the maximum. The coordinates are normalized to the distance $r$ between the loudspeaker and the microphone.

polar angles in the interval of $0^{\circ}$ to $30^{\circ}$ and $5 \mathrm{~cm}$ for polar angles in the interval of $32^{\circ}$ to $115^{\circ}$. As an example, the resulting directivity pattern at $20 \mathrm{kHz}$ of one microphone is shown in Figure 4. The highest sensitivity of the microphone was detected for small polar angles up to $15^{\circ}$. For polar angles up to $90^{\circ}$, the normalized sensitivity decreased up to $4 \mathrm{~dB}$, showing a radial symmetry. For larger polar angles up to $115^{\circ}$, the normalized sensitivity decreased up to $6 \mathrm{~dB}$. Additionally, regions with a decrease of up to $10 \mathrm{~dB}$ by insonifying the microphone from the back were detected. These positions correspond to the crossbeam of the microphone array.

\section{Ultrasound source}

To obtain a significant database on airborne workplace ultrasound for further research objectives, the object of investigation should preferably be a representative occupational source of airborne ultrasound. An ultrasonic welding machine was chosen, since ultrasonic welding is a widespread application with excitation frequencies that are representative of ultrasound techniques in industry and with noise emissions of high sound pressure levels. The welding machine was of the "HiQ SOLID SDM $20 \mathrm{kHz}$ " type made by Herrmann Ultraschalltechnik GmbH \& Co. KG. This type belongs to the "HiQ SOLID PropControl" series of tabletop devices, which is shown in Figure 5. The ultrasonic welding machine had outer dimensions of $643 \mathrm{~mm} \times$ $756 \mathrm{~mm} \times 1247 \mathrm{~mm}$ (width $\times$ depth $\times$ height $).$

To operate the welding machine in customized operating modes via remote control, it was necessary to supersede the "ULTRAPLAST SDM 2400" internal generator. For signal generation, an "ULTRABOND digital 48.20" external generator was used instead. This generator had a nominal working frequency of $20 \mathrm{kHz}$ and a nominal power of $4800 \mathrm{~W}$. The generator allowed single-pulsed, repetitively pulsed and continuous operation of the welding machine

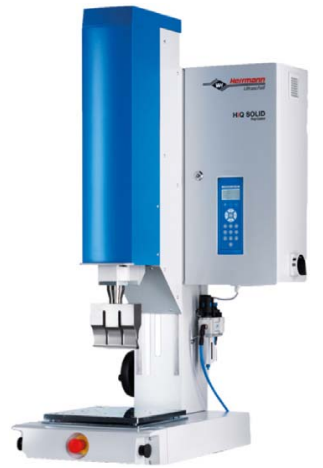

Figure 5. Ultrasonic welding machine of the "HiQ SOLID PropControl" series made by Herrmann Ultraschalltechnik $\mathrm{GmbH} \&$ Co. KG. A welding machine of this series, "HiQ SOLID SDM $20 \mathrm{kHz}$ " type, was used as the ultrasound source. Photograph courtesy of Herrmann Ultraschalltechnik GmbH \& Co. KG.

via remote control. All three operating modes are common for industrial applications depending on the intended purpose. Therefore, investigations using all of these operating modes were conducted. The generator was connected to a PC and controlled via the "DIASIM7" software program, which was provided by the manufacturer. The software program allowed the cycle and welding duration to be customized, as well as machine parameters such as the working frequency to be monitored and recorded online.

For the measurements, a cylindrical sonotrode was positioned approximately $15 \mathrm{~cm}$ above its counterpart on the platen. Preliminary investigations showed that multiple welding of the same workpiece leads to large variations of the working frequency. As replacing the workpiece after each welding process with the large number of measuring points intended was impracticable, the sonotrode was excited in air without a workpiece inserted to realize extensive measurements. Depending on the operating mode, the excitation of the sonotrode was started approximately $1-2 \mathrm{~h}$ in advance of each measurement. This settling time was necessary, as the excitation led to heating of the resonant unit and thereby to a frequency shift. The resonant unit was cooled with pressurized air through holes drilled in the converter connected to a closed pneumatic system to prevent it from overheating. An analysis of the performance data of the ultrasonic welding machine showed that the settling time chosen ensured that the variation of the working frequency in the steady state was smaller than the frequency resolution of the measurement. Thus, the ultrasonic welding machine can be assumed to be a suitable ultrasound source for extensive (and thereby long-lasting) measurements.

The ultrasonic welding machine was set up in the freefield environment on a table, which is representative of how this tabletop device is set up in industry. Since it was only possible to mount the microphone array in the $x y$-plane in the standard orientation, with the microphones aimed in the $-y$-direction, the welding machine had to be 


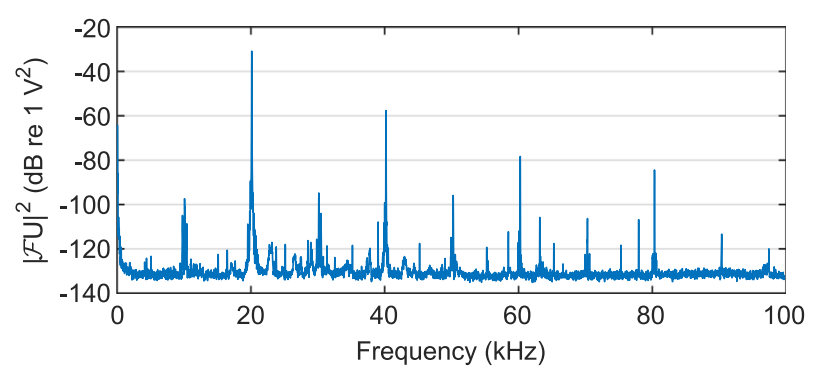

Figure 6. Square of the absolute value of the discrete Fourier transform of the signal $|\mathcal{F} U|^{2}$ of a single channel in the frequency range from $0 \mathrm{kHz}$ to $100 \mathrm{kHz}$ with a frequency resolution of $15.625 \mathrm{~Hz}$ time-averaged over $0.64 \mathrm{~s}$. For this measurement, the ultrasonic welding machine was operated in the continuous mode.

turned with the side under investigation facing the $y$-direction.

\section{Results}

The square of the absolute value of the discrete Fourier transform of the signal of a single channel is shown in Figure 6 as an example. The components of the signal corresponding to the working frequency of the ultrasonic welding machine at $20.1 \mathrm{kHz}$ can be clearly identified. Higher harmonics up to the third order and the subharmonic can be seen at $40.3 \mathrm{kHz}, 60.4 \mathrm{kHz}, 80.5 \mathrm{kHz}$ and $10.0 \mathrm{kHz}$ respectively. Estimating the noise floor to be $-130 \mathrm{~dB}\left(\mathrm{re} 1 \mathrm{~V}^{2}\right)$, the highest SNR is approximately $90 \mathrm{~dB}$ at the working frequency and the lowest SNR is approximately $30 \mathrm{~dB}$ at the frequency of the subharmonic.

The measurement result of a scan in a vertical plane $24 \mathrm{~cm}$ in front of the sonotrode is shown in Figure 7. The position of the plane close to the sonotrode was chosen to allow the sources of the sound field to be located. The measuring plane was $168 \mathrm{~cm}$ wide and $150 \mathrm{~cm}$ high. The map shows the $Z$-weighted equivalent continuous sound pressure levels time-averaged over $2 \mathrm{~s}$ of the one-third octave band with a center frequency of $20 \mathrm{kHz}$. A region with high sound pressure levels up to $127 \mathrm{~dB}$ is located in the center of the measuring plane. By comparing it with the corresponding coordinates, this region can be assigned to the sonotrode, which can thus be identified as the main source of the sound field. The sound field itself is characterized by a very finely structured speckle-like pattern. Regions of local sound pressure maxima are interspersed with spots of local sound pressure minima. In addition, a pattern of lines radiates from the central region. These linear structures may indicate reflections of ultrasonic waves emitted by the sonotrode at the workholding device, the platen and the base of the welding machine.

The analysis of the one-third octave band with a center frequency of $40 \mathrm{kHz}$ showed that the structure of the sound field at the first higher harmonic is even finer than the sound field at the working frequency. This applies to both the linear structures and the speckle-like areas. As indicated by Figure 6, the sound pressure levels of the one-third

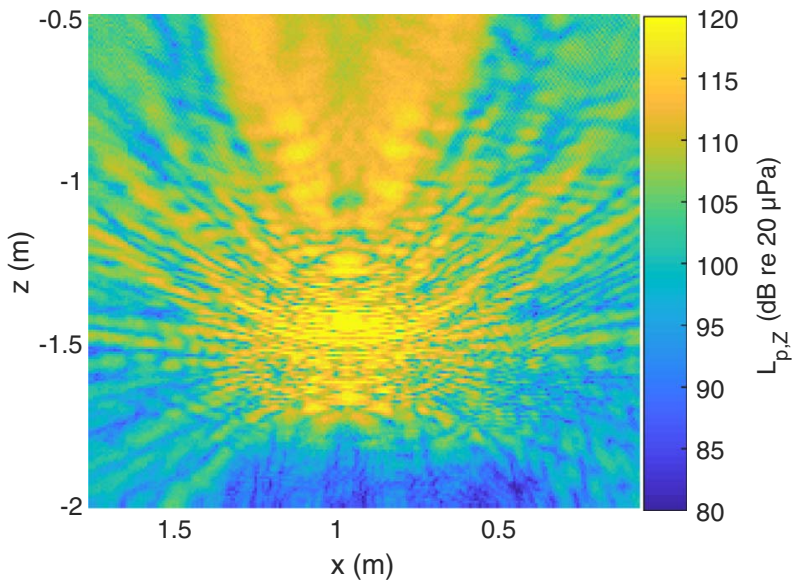

Figure 7. $Z$-weighted equivalent continuous sound pressure levels time-averaged over $2 \mathrm{~s} L_{p, Z \text {,eq } 2 \mathrm{~s}}$ of the one-third octave band with a center frequency of $20 \mathrm{kHz}$ in a vertical plane $24 \mathrm{~cm}$ in front of the sonotrode $\left(S_{\text {Figure } 7}: y=-0.80 \mathrm{~m}\right.$ ) with a spatial resolution of $1 \mathrm{~mm}$.

octave band with a center frequency of $40 \mathrm{kHz}$ are approximately 20-25 dB lower than the values at the working frequency. The analysis of the sound field of the one-third octave band with a center frequency of $10 \mathrm{kHz}$ showed that the structure of the sound field has a rougher, mostly linear structure. The considerably lower sound pressure levels compared to the values at the working frequency as indicated by Figure 6 are confirmed for the entire measuring plane.

Figure 8 shows the distribution of sound pressure levels in the one-third octave band with a center frequency of $20 \mathrm{kHz}$ in a vertical plane with the same dimensions as the measurement related to Figure 7 but with a distance of $99 \mathrm{~cm}$ in front of the sonotrode. Here, at a greater distance to the sonotrode, the sound field is still characterized by a heterogeneous distribution of local extrema of sound pressure levels.

The measurement result of a scan in a horizontal plane in front of the machine at a height of $111 \mathrm{~cm}$ is shown in Figure 9. The measuring plane was $168 \mathrm{~cm}$ wide and $75 \mathrm{~cm}$ deep. The sound field of the one-third octave band with a center frequency of $20 \mathrm{kHz}$ shows a radial characteristic with the region of the sonotrode as the origin in the middle of the top edge.

The measurement result of a scan at the back of the welding machine shown in Figure 10 indicates the strong influence of the shadowing effect. In addition, high sound pressure levels up to $124 \mathrm{~dB}$ were detected. Shadowing caused by the base of the welding machine can be seen at the lower edge of Figure 7. The shadow region is of the same width as the welding machine.

With the exception of Figure 10, all sound fields presented have mirror symmetry. The mirror plane is $S_{v}$ : $x=0.981 \mathrm{~m}$, which incorporates the sonotrode. The sound fields shown in Figures 7 and 10 indicate another mirror plane perpendicular to $S_{v}$. This additional mirror plane is $S_{h}: z=-1.52 \mathrm{~m}$, which is near the tip of the sonotrode. 


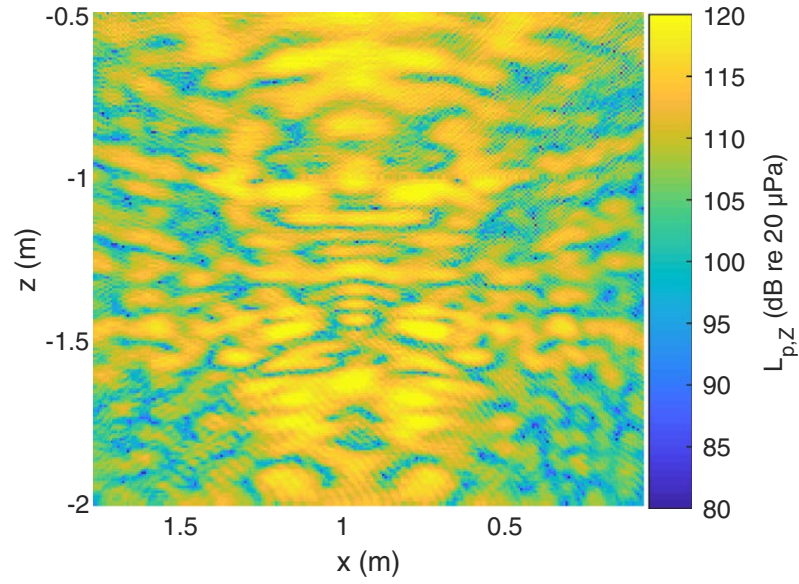

Figure 8. Z-weighted equivalent continuous sound pressure levels time-averaged over $0.64 \mathrm{~s} L_{p, Z \text { eq }} 0.64 \mathrm{~s}$ of the one-third octave band with a center frequency of $20 \mathrm{kHz}$ in a vertical plane $99 \mathrm{~cm}$ in front of the sonotrode $\left(S_{\text {Figure } 8}: y=-0.05 \mathrm{~m}\right)$ with a spatial resolution of $0.6 \mathrm{~mm}$.

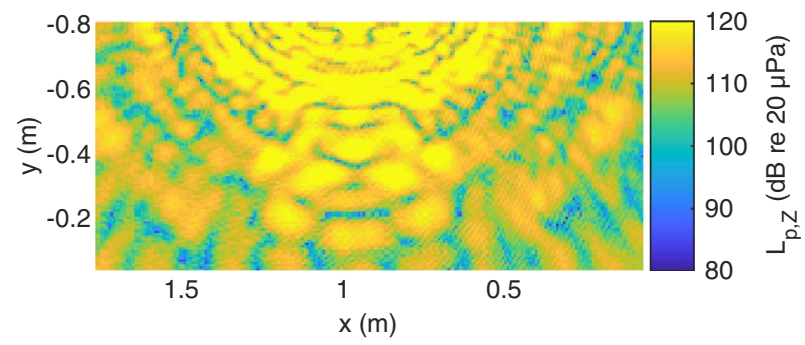

Figure 9. Z-weighted equivalent continuous sound pressure levels time-averaged over $0.64 \mathrm{~s} L_{p, Z \text { eeq } 0.64 \mathrm{~s}}$ of the one-third octave band with a center frequency of $20 \mathrm{kHz}$ in a horizontal plane $24 \mathrm{~cm}$ to $99 \mathrm{~cm}$ in front of the sonotrode at a height of $1.11 \mathrm{~m}$ ( $S_{\text {Figure } 9}: z=-1.26 \mathrm{~m}$ ) with a spatial resolution of $0.6 \mathrm{~mm}$.

For the measurement associated with Figure 7, the ultrasonic welding machine was operated in the repetitively pulsed mode with a cycle duration of $2 \mathrm{~s}$ and an excitation pulse with a welding duration of $0.5 \mathrm{~s}$. The measurements associated with Figures 8-10 were carried out by operating the ultrasonic welding machine in the continuous mode. A comparison of the measurement results for the repetitively pulsed mode and the continuous mode showed the same structure of the sound field but with reduced sound pressure levels for the repetitively pulsed mode of approximately $8 \mathrm{~dB}$.

Measurements at the front of, back of, left side of and from above the welding machine with up to 252000 measuring points and a total measuring time of up to 6 days and $14 \mathrm{~h}$ were performed using spatial resolutions in the range from $6 \mathrm{~mm}$ to $0.1 \mathrm{~mm}$. The highest $Z$-weighted equivalent continuous sound pressure level time-averaged over $0.64 \mathrm{~s} L_{p, Z \text {,eq } 0.64 \text { s }}$ of the one-third octave band with a center frequency of $20 \mathrm{kHz}$ was $138 \mathrm{~dB}$.

For reasons explained in Section 4, the sound field of the ultrasonic welding machine was measured with no

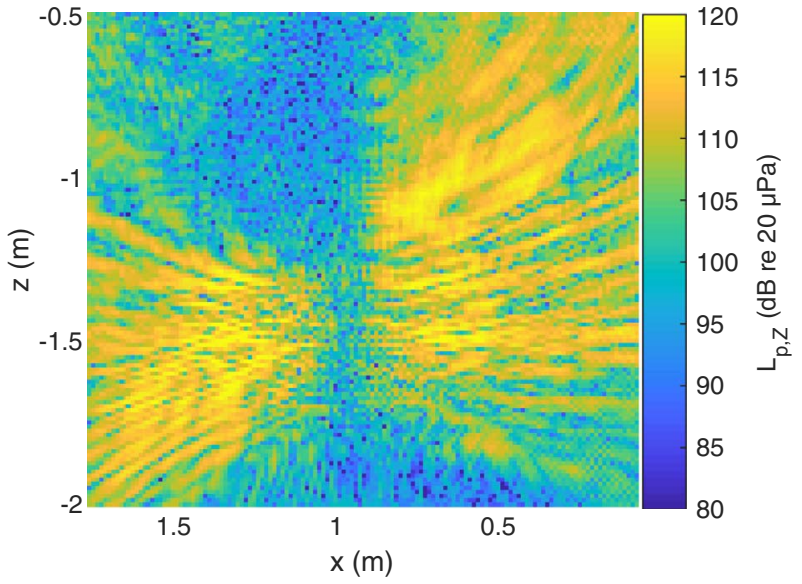

Figure 10. Z-weighted equivalent continuous sound pressure levels time-averaged over $0.64 \mathrm{~s} L_{p, Z, \text { eq }} 0.64 \mathrm{~s}$ of the one-third octave band with a center frequency of $20 \mathrm{kHz}$ in a vertical plane at the back side with a distance of $59 \mathrm{~cm}$ to the sonotrode $\left(S_{\text {Figure 10 }}: y=-0.75 \mathrm{~m}\right)$ with a spatial resolution of $0.5 \mathrm{~mm}$.

workpiece inserted and while exciting the sonotrode in air. To complement the results described so far, measurements using a single microphone system were conducted at a single measuring point for the two operating modes while exciting the sonotrode in air and while welding a workpiece. For these measurements, the microphone was fixed on a tripod $57 \mathrm{~cm}$ centrally in front of the sonotrode at a height of $1.34 \mathrm{~m}$. The measuring point had the coordinates $(0.98 \mathrm{~m},-0.46 \mathrm{~m},-1.03 \mathrm{~m})$. The audio signal was sampled with a sample frequency of $192 \mathrm{kHz}$. Further processing of the measuring data, which was stored as WAVE files, was performed as an offline analysis. The averaged Z-weighted equivalent continuous sound pressure levels time-averaged over $2 \mathrm{~s}$ were $120.6 \mathrm{~dB}$ for the continuous mode, $113.3 \mathrm{~dB}$ for the repetitively pulsed mode and $106.8 \mathrm{~dB}$ for welding a workpiece. In Figure 11, the $Z$-weighted equivalent continuous sound pressure levels are shown as a spectrogram with a frequency resolution of $250 \mathrm{~Hz}$ and a periodogram with a frequency resolution of $15.625 \mathrm{~Hz}$ for measurements of the three operating modes. The measuring duration was $2 \mathrm{~s}$ for each measurement. Compared to the periodogram of the measurement of the continuous mode, shown in Figure 11b, the periodogram of the measurement of the repetitively pulsed mode, shown in Figure 11d, is characterized by increased sound pressure levels of components in the frequency interval from $20 \mathrm{kHz}$ to $40 \mathrm{kHz}$ and decreased sound pressure levels at the fundamental frequency, the subharmonic and the higher harmonics. The corresponding spectrogram, which is shown in Figure 11c, indicates the beginning of the welding pulse at $0.6 \mathrm{~s}$ and a broadband noise signal after $1.1 \mathrm{~s}$, when the welding pulse ends after a duration of $0.5 \mathrm{~s}$. It can be seen that the decay of the signal at the fundamental frequency takes another $0.5 \mathrm{~s}$. A similar characteristic can be identified in the spectrogram of the measurement of the welding process, shown in Figure 11e. However, additional tonal components in the frequency interval from 


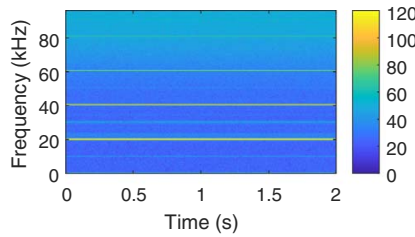

(a)

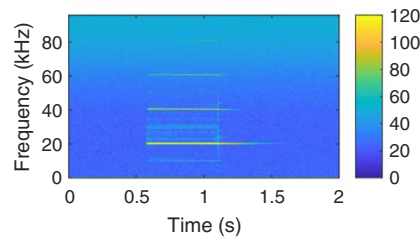

(c)

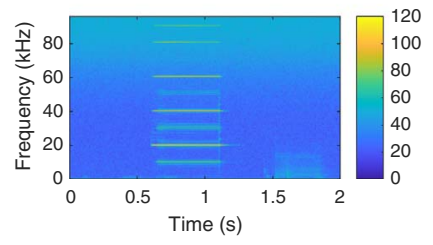

(e)

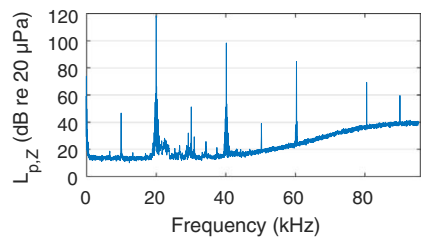

(b)

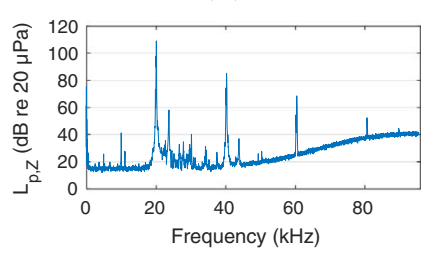

(d)

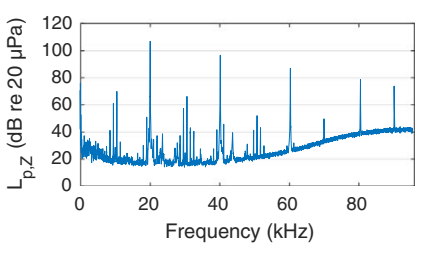

(f)
Figure 11. (a, c, and e) Spectrograms and (b, d, and f) periodograms of measurements with a duration of $2 \mathrm{~s}$. Zweighted equivalent continuous sound pressure levels $L_{p, Z \text {,eq } 2 \mathrm{~s}}$ ( $a$ and b) for the continuous mode, ( $c$ and d) for the repetitively pulsed mode and (e and f) for welding a workpiece.

$5 \mathrm{kHz}$ to $55 \mathrm{kHz}$ can be identified. Specifically, the sound pressure levels at the subharmonic $(10 \mathrm{kHz})$ and the ultraharmonics $(30 \mathrm{kHz}$ and $50 \mathrm{kHz})$ increase noticeably compared to the other operating modes.

For an interpretation of the sound field structure generated, the length of the near field along the acoustic axis was calculated. The length of the near field is given by the distance of the sound pressure maximum furthest from the sound source before the sound pressure monotonically decreases. For a piston mounted in an infinite rigid baffle, the distance $l$ of this maximum is given by:

$$
l=\frac{R^{2}}{\lambda}-\frac{\lambda}{4}
$$

in which $R$ is the radius of the piston and $\lambda$ is the wavelength of the emitted sound ([24], p. 201). Hence, the length of the near field of the sonotrode with a radius $r=1.5 \mathrm{~cm}$, as used in this study, is approximately $0.9 \mathrm{~cm}$ at the working frequency of $20.1 \mathrm{kHz}$.

For a quantitative description of the sound field structure, the sound field shown in Figure 7 was analyzed using methods of digital image processing. The coordinates of local sound pressure minima and maxima with a prominence of at least $6 \mathrm{~dB}$ were determined using the Fiji image processing program (2.0.0-rc-69/1.52p, 64-bit). In this way, 361 local sound pressure extrema were determined. As shown in Figure 12, the median distance between a local sound pressure maximum and its closest local sound pressure minimum is $2.6 \mathrm{~cm}$. On average, the difference between

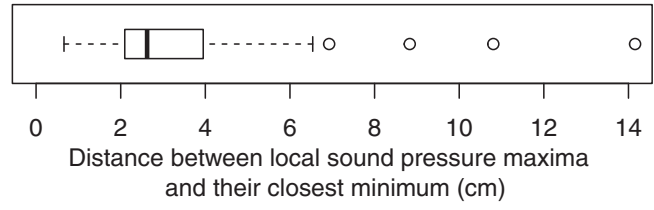

Figure 12. Distance of local sound pressure maxima to their closest local sound pressure minimum. Analysis of Figure 7.

local sound pressure minima and maxima is approximately $20 \mathrm{~dB}$.

\section{Measurement uncertainty}

The uncertainty of the linear step size of the scanning unit was $\pm 1.25 \mu \mathrm{m}[18]$. The measurement repeatability of the reference point of the scanning unit was $\pm 20 \mu \mathrm{m}$ per axis ([20], p. B-38). The worst-case process loss due to the Hann window is $3.18 \mathrm{~dB}[25]$. The uncertainty of the calibrated free-field sensitivity is less than or equal to $0.8 \mathrm{~dB}$. The uncertainty due to the directivity pattern varies depending on the incidence angle of the sound. Assuming that the sound emitted or reflected in the region of the sonotrode is the main source, the uncertainty depends on the distance of the measuring plane to the sonotrode and on the distance of the measuring point to the center of the measuring plane. The larger the incidence angle is, the lower the sensitivity of the microphone is. Therefore, the signal at measuring points in the peripheral area of the measuring plane was underrated. The largest incidence angles of approximately $75^{\circ}$ occurred at the measurement related to Figure 7. The corresponding decrease in sensitivity is approximately $3 \mathrm{~dB}$. The total uncertainty of the measurement related to Figure 7 varies between $1.3 \mathrm{~dB}$ for measuring points in the central region of the measuring plane (incidence angles of approximately $0^{\circ}$ ) and $3.3 \mathrm{~dB}$ for measuring points in the peripheral area (incidence angles of approximately $75^{\circ}$ ).

\section{Discussion}

The purpose of this study was to investigate the structure of airborne ultrasound fields for occupational safety and health. To this end, technical and procedural requirements concerning spatial resolution, frequency range, dynamic range, SNR and traceability for a measuring system for airborne ultrasound fields were formulated. Based on these factors, a measuring system was set up, calibrated and used for extensive measurements of the airborne ultrasound field of an ultrasonic welding machine. Selecting components that fulfilled the formulated requirements was possible only with great difficulty due to limited data and standards concerning the ultrasonic frequency range. Manufacturer data and measurement procedures based on quantities and definitions that were created for the audible frequency range did not always allow conclusions to be drawn for the ultrasonic frequency range. Where data 
was missing, measurements had to be performed to calibrate and thereby evaluate the measuring instruments. Standardized measurement procedures and experimental setups are the subject of current research to extend the existing capabilities from the audible to the ultrasonic frequency range. The calibration of the frequency response and supplemental measurements as well as the measurement results achieved in this study confirm that the measuring system set up has successfully fulfilled the formulated requirements. One requirement concerning the effective radius of the microphone diaphragm, which was adapted from IEC $62127-1$, could not be fulfilled for the entire frequency range. However, the strict criterion formulated for the effective radius was satisfied for frequencies up to $29 \mathrm{kHz}$, which includes the entire one-third octave band with a center frequency of $20 \mathrm{kHz}$. All sound field maps shown in this paper are analyses of the one-third octave band with a center frequency of $20 \mathrm{kHz}$. The directivity patterns of the microphones used in the measuring system were investigated. Since the directions of the sound measured with the microphones are unknown, a correction could not be applied. However, the corresponding contribution to the measurement uncertainty was estimated. Considering the free-field environment in the scanner unit, it may be reasonable to assume that sound arriving from the back of the microphone can be neglected. Therefore, the decreased and partially inhomogeneous sensitivity of the microphones for incidence angles larger than $90^{\circ}$ are not a significant drawback. An analysis of the periodogram shown in Figure 6 confirmed that the high SNRs required were achieved. The measuring system enabled three-dimensional scanning measurements of sound pressures of airborne ultrasound fields. The setup enabled measurements with a very high spatial resolution of $0.1 \mathrm{~mm}$. The large volume of the freefield environment of approximately $8 \mathrm{~m}^{3}$ enabled the investigation of large ultrasound sources and setups with additional equipment such as an artificial head and objects acting as reflecting surfaces.

The findings support the assumption that ultrasound fields produced by industrial sources contain a complex distribution of sound pressure levels. An analysis of the sound field shown in Figure 7 using digital image processing revealed that the median distance between local sound pressure maxima and their closest local sound pressure minimum was only $2.6 \mathrm{~cm}$. It must be considered that these distances may be even smaller for higher frequencies. The difference between these local sound pressure extrema is $20 \mathrm{~dB}$. However, larger differences up to $40 \mathrm{~dB}$ were found. Here, it is apparent that measurements at a single point are not representative for the whole ultrasound field. Likewise, determining the exposure to airborne ultrasound based on static measurements at a single point does not provide reliable results. For this reason, a novel measurement procedure adapted to the specifics of airborne ultrasound is needed.

The structures of the ultrasound field cannot be explained as a consequence of the near field of the sonotrode. Since the radius of the sonotrode is still smaller than the wavelength of the sound emitted at the working frequency, the ratio of the radius and the wavelength is small $(R / \lambda=0.9)$, as is the length of the near field $(l=0.9 \mathrm{~cm})$. Moreover, it must be considered that the measuring planes were parallel to the acoustic axis on which the length of the near field was defined. Thus, it was not possible to make a statement on the length of the near field in terms of a distance between the sonotrode and the measuring plane. The structure of the ultrasound field may be determined via the following two effects. On the one hand, the sonotrode emitting direct ultrasound in combination with reflections of ultrasound at parts of the welding machine may form a system consisting of a source and a mirror source, enlarging the effective size of the ultrasound source of sound waves propagating in the direction of the measuring plane. Since the sonotrode was positioned $15 \mathrm{~cm}$ above the platen, the estimated distance between the source and the mirror source was $30 \mathrm{~cm}$. Using equation (1), the length of the near field of a source with an effective radius of $15 \mathrm{~cm}$ is approximately $1.3 \mathrm{~m}$. This could explain the extended interference patterns in Figures 8 and 9, which show near-field characteristics as expected for such very large sources. On the other hand, it must be considered that the far field of a piston is formed by a main lobe and several side lobes. It must be assumed that the sound of these lobes is reflected at multipartite reflecting surfaces such as the workholding device, the platen and the base of the welding machine. Considering that the sound beam broadens at a certain aperture angle, the reflections of the lobes may further complicate the sound field structure by forming the starburst pattern of lines radiating from the central region as shown in Figures 7 and 10. Consequently, the $v$-shaped region at the upper border in Figure 7 may be assigned to the main lobe and the linear structures may be assigned to the side lobes. In this way, the radial structures of Figure 9 may be explained as well.

Measurements at the back of the ultrasonic welding machine revealed a strong shadowing effect. This effect is caused by obstacles in the propagation path of ultrasonic waves if these obstacles are geometrically large in relation to the wavelength of the propagating ultrasound. As a result, the sound pressure levels are significantly lower in the shadows of these obstacles. The results of measurements performed at the front of, back of, left side of and from above the ultrasonic welding machine indicate that ultrasound with high sound pressure levels is emitted in all directions. Workers nearby may be exposed to these emissions from the primary source directly or via reflections from the ceiling, floor or other surfaces.

For the measurements performed, it was necessary to operate the ultrasonic welding machine with no workpiece inserted and while exciting the sonotrode in air. Consequently, the sound fields presented in the results will differ from sound fields that occur during welding of a workpiece. However, the purpose was not to assess the exact ultrasound field of this machine that arose during the welding process. The aim was instead to obtain initial insights of ultrasonic fields arising in an occupational context in general and testing the measuring system. Moreover, both the precise structure and the sound pressure levels of the 
ultrasound field strongly depend on various influencing factors, e.g. the shape and size of the sonotrode, the workpiece, the surrounding area, obstacles or persons in the sound field, the operating mode and the ultrasonic modality. Consequently, every conceivable measurement setup or configuration merely represents a unique case with limited potential for generalization. It should be noted that the setup investigated is not a worst-case scenario. Although the measuring results show a pronounced, finely structured ultrasound field, setups with larger ratios of the radius of the sound source to the wavelength of the emitted sound are common. It can be assumed that such setups produce further complicated sound field structures. Although the investigations were conducted to acquire a data basis for further research and development of a measurement procedure for occupational safety and health that can be used to assess the exposure of workers to airborne ultrasound, the measuring system used here could be used for various application scenarios, e.g. development and improvement of transducers, sensors and acoustic materials or assessment of the emission of ultrasound sources.

To show the influence of the different operating modes, measurements with a single microphone system were performed at one measuring point. As the sonotrode was excited in the repetitively pulsed mode for $0.5 \mathrm{~s}$ in a cycle duration of $2 \mathrm{~s}$, the expected decrease in the sound pressure level compared to the value of the continuous mode is $6 \mathrm{~dB}$. The difference $(7.3 \mathrm{~dB})$ between the sound pressure levels measured for both modes was a good approximation of this decrease. The deviation from the expected value may be caused by transient oscillation effects at the beginning and end of the excitation pulse. The periodogram of the measurement of welding a workpiece at this measuring point indicates increased sound pressure levels at the subharmonic and ultraharmonics compared to the other operating modes. Nevertheless, the tonal characteristic of the periodogram is still preserved. A comparison of measurements of the sound field for the continuous operating mode and the repetitively pulsed mode showed a high degree of similarity of the structures. Based on the findings of measurements at a single point, it can be assumed that the structure of the sound field while welding a workpiece also has substantial similarity to the aforementioned cases. While a general statement for the rest of the sound field cannot be made, the findings provide further support for the hypothesis that the ultrasound field is strongly affected by the concrete conditions of emission. For a workpiece under ultrasonic welding, opposite effects such as shadowing of the sonotrode and an increase in the surface of the emitting source have to be considered with all resulting implications.

\section{Conclusion and outlook}

In this work, technical and procedural requirements for a measuring system for airborne ultrasound have been derived. Subsequently, a measuring system for threedimensional high spatial resolution scans of airborne ultrasound fields has been set up and calibrated successfully. Extensive measurements of the airborne ultrasound field of an ultrasonic welding machine have been conducted. They have demonstrated that the measuring system fulfills the relevant requirements and is thereby able to characterize airborne ultrasound fields using traceable measurement results. The measurements conducted confirmed the assumptions made at the beginning and revealed a complex and very finely structured sound field and (in some cases) very high sound pressure levels.

While current limitations led to the choice of $1 / 4^{\prime \prime}$ microphone cartridges for the measuring system, in the future, testing of $1 / 8^{\prime \prime}$ microphones or different microphone types concerning their suitability is recommended. The modular concept of the measuring system allows an easy exchange of single components. A smaller sensor is still preferable, as the diameter of the microphone currently limits the spatial resolution of the measuring system. An enhancement by adding further motors to realize at least two rotational degrees of freedom for the microphones is conceivable. This would allow more flexible setups and measuring paths, offer the possibility to measure sound power levels and might reduce the influence of the directivity pattern of the microphones. A modified microphone array with non-equidistant arrangement of the microphones could be used to trim the spatial resolution independently of the scanning unit's capability. The number of microphone channels could be increased to acquire data at more measuring points simultaneously and thereby reduce the total measuring time. This would help to overcome the current limitation of very long measuring times. A reduced total measuring time would enable the investigation of ultrasonic sources that can be operated in a steady state only for a short period.

Further research on the influence of objects and persons on the ultrasound field is advisable. The results support the need for the development of a measurement procedure adapted to the specifics of airborne ultrasound, as it is apparent that assumptions made for measurement procedures in the audible frequency range do not comply with the characteristics of ultrasound fields. It is also apparent that individual measuring points are not representative of the highly heterogeneous spatial distribution of sound pressure levels. Since methods of digital image processing have led to positive initial results, these methods should be applied in further approaches concerning the quantification of the structure of ultrasound fields. It is impossible for those working in occupational safety and health to investigate workplaces in as much detail as with the measuring system developed here for the laboratory. Therefore, the results of the measurements performed will be the basis for the development of a measurement procedure that will allow the exposure of a worker to airborne workplace ultrasound to be measured with less time and effort and fewer measuring instruments.

\section{Conflict of interest}

Author declared no conflict of interests. 


\section{Acknowledgments}

This project has received funding from the EMPIR programme co-financed by the Participating States and from the European Union's Horizon 2020 research and innovation programme. The contents of this article reflect only the authors' views. EURAMET is not responsible for any use that may be made of the information this article contains. We gratefully acknowledge the support of the Braunschweig International Graduate School of Metrology (B-IGSM). We would like to thank Herrmann Ultraschalltechnik GmbH \& Co. KG for providing an ultrasonic welding machine. We would like to offer special thanks to Andreas Schmidt, Jens Wieczorek and Sascha Fabian for the technical assistance and to Sonja Walther and Thomas Postma for assistance in collecting the data.

\section{References}

1.T.G. Leighton: Are some people suffering as a result of increasing mass exposure of the public to ultrasound in air? Proceedings of the Royal Society A: Mathematical, Physical and Engineering Science 472, 2185 (2016). Article ID 20150624. https://doi.org/10.1098/rspa.2015.0624.

2. B.W. Lawton: Damage to Human Hearing by Airborne Sound of Very High Frequency or Ultrasonic Frequency. Contract Research Report 343. HSE Books, University of Southampton and Health \& Safety Executive, Sudbury, Suffolk CO10 2WA, England, United Kingdom, 2001.

3. Physikalisch-Technische Bundesanstalt, Editor: EMPIR 15HLT03 Ears II - Metrology for Modern Hearing Assessment and Protecting Public Health from Emerging Noise Sources. Project Website. 2016. URL: http://www.earsproject.eu/ (Visited on 09/07/2019).

4. G. Harvey, A. Gachagan, T. Mutasa: Review of highpower ultrasound-industrial applications and measurement methods. IEEE Transactions on Ultrasonics, Ferroelectrics, and Frequency Control 61, 3 (2014) 481-495. https://doi. org/10.1109/tuffc.2014.2932.

5. A. Holm, H.W. Persson: Optical diffraction tomography applied to airborne ultrasound. Ultrasonics 31, 4 (1993) 259265. https://doi.org/10.1016/0041-624x(93)90019-v.

6. O.B. Matar, C. Rossignol, L. Pizarro, S.D. Santos, F. Patat: Mapping of airborne ultrasonic fields using optical heterodyne probing and tomography reconstruction, in 2000 IEEE Ultrasonics Symposium. Proceedings. An International Symposium (Cat. No. 00CH37121) (Oct. 22, 2000), IEEE. 2000. https://doi.org/10.1109/ultsym.2000.921521.

7. R. Schöneweiß, C. Kling, C. Koch: Datasets of High Spatial Resolution Scans of the Airborne Ultrasound Field at the Front, Back and Left Side of an Ultrasonic Welding Machine. Dataset. Version 1.0.0. Zenodo, 2020. https://doi.org/ 10.5281 /zenodo.3550527.
8. R. Schöneweiß, C. Kling, C. Koch: Free-Field Sensitivity of Four Electro-Acoustic Measuring Chains at $0^{\circ}$ Incidence Angle in the Frequency Range $0.25 \mathrm{kHz}$ to $100 \mathrm{kHz}$. Dataset. Version 1.0.0. Zenodo, 2020. https://doi.org/10.5281/zenodo.3552412.

9. H. Kuttruff: Room Acoustics. 5th ed. Spon Press, 2009.

10. L.B. Evans, H.E. Bass, L.C. Sutherland: Atmospheric absorption of sound: Theoretical predictions. The Journal of the Acoustical Society of America 51, 5B (1972) 15651575. https://doi.org/10.1121/1.1913000.

11. C. Ullisch-Nelken, H. Kusserow, A. Wolff: Analysis of the noise exposure and the distribution of machine types at ultrasound related industrial workplaces in Germany. Acta Acustica United With Acustica 104, 5 (2018) 733-736. https://doi.org/10.3813/aaa.919212.

12. International Electrotechnical Commission (IEC): IEC 62127-1: 2007-08+COR1:2008+AMD1:2013-02 CSV (Consolidated Version) - Ultrasonics - Hydrophones - Part 1: Measurement and Characterization of Medical Ultrasonic Fields up to $40 \mathrm{MHz}$. Norm. International Electrotechnical Commission (IEC), 2013.

13. A. Wolff, C. Ullisch-Nelken, R. Schöneweiß, C. Kling, H. Kusserow, M. Fletcher, B. Lineton, C. Koch: Noise Exposure at Ultrasound Related Industrial Workplaces and Public Sites. Dataset. Version 1.0.0. 2019. https://doi.org/10.5281/ zenodo.3163215.

14. K.F. Herzfeld, T.A. Litovitz: Absorption and dispersion of ultrasonic waves, in Pure and Applied Physics, Vol. 7, Academic Press, New York, NY [u.a.]. 1959.

15. H.E. Bass, F.D. Shields: Absorption of sound in air: Highfrequency measurements. The Journal of the Acoustical Society of America 62, 3 (1977) 571-576. https://doi.org/ 10.1121/1.381576.

16. H.E. Bass, L.C. Sutherland, A.J. Zuckerwar, D.T. Blackstock, D.M. Hester: Atmospheric absorption of sound: Further developments. The Journal of the Acoustical Society of America 97, 1 (1995) 680-683. https://doi.org/10.1121/ 1.412989

17. H.E. Bass, L.C. Sutherland, A.J. Zuckerwar, D.T. Blackstock, D.M. Hester: Erratum: Atmospheric absorption of sound: Further developments [J. Acoust. Soc. Am. 97, 680 683 (1995)]. The Journal of the Acoustical Society of America 99, 2 (1996) 1259-1259. https://doi.org/10.1121/1.415223.

18. Isel Germany AG: MS 135 HT-2 - MS 200 HT-2. Eichenzell, Germany, 2010.

19. Isel Germany AG: Linear Units with Spindle Drive/LES4 LES5 - LES6. Eichenzell, Germany, 2018.

20. Isel Germany AG: Automation. Eichenzell, Germany, 2017.

21. GRAS Sound \& Vibration A/S: Product Information GRAS 40BF. 2019.

22. GRAS Sound \& Vibration A/S: Product Information GRAS 40DP. 2019.

23. GRAS Sound \& Vibration A/S: Product Information GRAS 12AQ. 2019.

24. V.A. Šutilov: Physik des Ultraschalls. Ed. and Trans. Russian by P. Hauptmann. Akademie-Verlag, Berlin, 1984.

25. F.J. Harris: On the use of windows for harmonic analysis with the discrete Fourier transform. Proceedings of the IEEE 66, 1 (1978) 51-83. https://doi.org/10.1109/proc.1978.10837.

Cite this article as: Schöneweiß R, Kling C \& Koch C. 2020. A laboratory study for occupational safety and health on the structure of airborne ultrasound fields. Acta Acustica, 4, 12. 